\title{
Factores contextuales de la migración de retorno a los municipios de las Zonas Metropolitanas del Estado de México 2010-2015*
}

\section{Contextual factors of return migration to the municipalities of the metropolitan areas of the State of Mexico 2010-2015}

\author{
Juan Gabino González-Becerril y Eduardo Andrés Sandoval-Forero \\ Centro de Investigación y Estudios Avanzados de la Población/ \\ Universidad Autónoma del Estado de México, México
}

\section{Resumen}

Este texto tiene como objetivo mostrar la orientación de la migración de retorno al Estado de México, principalmente desde Estados Unidos entre 2010 y 2015. Con esto se busca probar que la intensidad migratoria juega un papel fundamental en la orientación de la migración de retorno hacia municipios metropolitanos, y no hacia municipios rurales que también son determinados por el nivel de pobreza y la marginación. Para sostener nuestro argumento se usaron datos del índice de marginación municipal del CONAPO de 2010-2015, la información sobre la pobreza estimado por el CONEVAL también a nivel municipal y el Índice de Marginación (2010-2015) estimado por el CONAPO, a la vez que apoyamos estos resultados con el modelo de correspondencia múltiple para probar el agrupamiento de la migración de retorno a nivel municipal.

Palabras clave: Migración de retorno, intensidad migratoria, pobreza, marginación, correspondencia múltiple.

\section{Abstract}

This text aims to show the orientation of return migration to the State of Mexico, mainly from the United States between 2010 and 2015. This seeks to prove that migration intensity plays a fundamental role in the orientation of return migration to municipalities metropolitan, and not so towards rural municipalities that are also determined by the level of poverty and marginalization. To support our argument, data from the 2010-2015 CONAPO municipal marginalization index, the poverty information estimated by CONEVAL also at the municipal level and the Marginalization Index (2010-2015) estimated by CONAPO were used at the same time We support these results with the multiple correspondence model to test the grouping of return migration at the municipal level.

Keywords: Return migration, migratory intensity, poverty, marginalization, multiple correspondence.

* Este artículo es un producto derivado del proyecto "Obstáculos de la incorporación a la educación básica, media superior y superior de la población migrante retornada al Estado de México, 2015", con registro ante la Secretaría de Investigación y Estudios Avanzados de la Universidad Autónoma del Estado de México con clave 4806/2019CIB. 


\section{INTRODUCCIÓN}

D urante la última década del siglo XX y la primera del nuevo milenio, la migración internacional mexiquense se ha incorporado de manera plena en la migración internacional, específicamente a Estados Unidos de América (E.E.U.U), dando cuenta de ello el Censo de Población y Vivienda del 2010, el cual colocó al Estado de México en el cuarto lugar de estados expulsores de migrantes recientes a E.E.U.U, con 67 mil 595 emigrantes con destino al vecino país del norte, esto significa que participa de cambios en el volumen, tendencias, modalidades y características sociodemográficas de los migrantes internacionales. En este sentido, una premisa ha sido el incremento de los flujos de migrantes mexiquenses y sus familiares que se van, pero se destaca de quienes retornan desde Estados Unidos después de haber vivido y trabajado durante un largo tiempo en dicho país.

La migración de retorno no es un fenómeno nuevo, pero sí una preocupación reciente en la investigación y la política gubernamental, porque presenta rasgos que la distinguen de los flujos migratorios del pasado. Los retornos que se registran en este texto son reconocidos como voluntarios en el contexto de la crisis económica de 2008 y la implementación de políticas restrictivas en materia migratoria en Estados Unidos, que son algunos de los factores que han incentivado los flujos de retorno en los últimos años (Ramírez y Aguado 2013). Además se debe considerar que el contexto económico y político está en constante cambio, y en materia de migración de retorno ha mostrado una particular sensibilidad a estos cambios en las últimas décadas, por ejemplo, se está generando expectativa respecto al impacto en el retorno después de que Donald Trump asumiera en enero de 2017 la presidencia de E.E.U.U quien de manera abierta ha expresado una postura política antiinmigrante, particularmente hacia los mexicanos, por lo que se prevé incentivará un retorno impulsado por el miedo y, en palabras de Massey (2016), provocará un incremento en las deportaciones al interior del país.

Ante estas premisas, el trabajo está organizado de la siguiente manera: exponemos los enfoques que exponen los factores de la migración de retorno, acto seguido exponemos los antecedentes sobre la migración de retorno a México y la del Estado de México; y por último el análisis de los factores que inhiben o atrae a los retornados, los cuales hemos denominado 
Variables de contexto de llegada del migrante retornado y su familia. Dichas variables son examinadas con base en el modelo de correspondencia múltiple cuya técnica permite agrupar a los municipios metropolitanos y rurales según atracción y rechazo.

\section{ENFOQUE ACERCA DE LOS FACTORES DETERMINANTES DE LA MIGRACIÓN DE RETORNO}

Los estudios migratorios, y en específico los de retorno, han sido referencia desde los pioneros como Ravenstein, entre 1885 y 1889 , y los estudios de la migración de retorno con científicos sociales europeos como Sjaastad (1962); Appleyard (1962); Goldstein (1964); Richmond (1983), entre otros. Pero lo que llama aún más la atención es un texto de Fernández Guzmán (2011), el cual expone una síntesis de los trabajos en torno a los enfoques de migración de retorno. Dicho autor sostiene que hay dos líneas de investigación acerca de este tema: una de carácter cualitativo y otra de carácter cuantitativo, y que, sin embargo, es mucho lo que se tiene que investigar sobre la dimensión histórica, antropológica, sociológica, psicológica, económica, demográfica o política que trae consigo este movimiento al lugar de salida.

El fenómeno del retorno tiene diversas perspectivas desde las cuales puede ser analizado; a manera de síntesis se puede señalar que son tres grandes corrientes: la primera hace referencia al retorno desde un contexto económico en el que el proceso de toma de la decisión de regresar por parte del migrante se da en función al éxito o fracaso en términos monetarios. Una segunda corriente es aquella que ve al retorno como respuesta, o en función de las redes sociales y transnacionalismo; en este caso el migrante decide regresar por melancolía, porque conservó sus redes sociales o por cuestiones familiares; aquí encaja la teoría de la causalidad acumulativa, la teoría de las redes sociales y el enfoque transnacional. Por último, tenemos la tercera corriente: el retorno involuntario o forzado, en el cual el migrante se ve obligado a retornar, por ejemplo, con las deportaciones y repatriaciones, o bien, los retornos en los cuales el migrante es obligado políticamente o forzado a regresar de manera voluntaria para evitar ser sometido a las regulaciones inmigratorias estadounidenses, pero en realidad no por convicción del propio migrante. Para esta investigación nos interesa rescatar las aportaciones acerca de los factores de contexto que sesgan la migración de retorno en general y de manera puntual en su lugar de llegada.

Seguimos el trabajo de Lee (1974), quien se centra en el migrante retornado y examina las relaciones con variables demográficas básicas (edad, 
sexo, raza), y que también pone atención en los factores de localización. En esta misma línea y por esos tiempos, William A. Glaser y Christopher Habers (1974, citado en Fernández Guzmán, 2011) identifican los diferentes motivos económicos y sociales para decidir quedarse (ingreso, igualdad de empleo, nuevos desarrollos en el campo profesional y la existencia de empleos más atractivos) o retornar (familia, amigos, sentido patriótico, discriminación étnica y racial).

Nina Toren (1976) examina las relaciones entre ciertas características y motivaciones del retorno migrante de Estados Unidos a Israel. Utilizando el modelo push-pull, afirma que los motivos para retornar son predominantemente del orden pull (atracción por el lugar de origen).

Chapman y Mansell (1983), en su análisis sobre la migración internacional, se centran en la circulación o flujos recíprocos de gente, con específica referencia a las sociedades del tercer mundo. Dichos autores sostienen que para comprender completamente la complejidad de la circulación de la migración debe ser analizada en varias escalas: la micro (individual-familiar), la meso (comunidad, sistema de población, región) y la macro (país, continente, mundo). Asimismo, sugieren que se deben enfocar con mucha atención en las estructuras sociales, políticas y económicas, las cuales atan e inciden recíprocamente en los flujos de ida y regreso.

Por su parte, Lockwood (1990) analiza el impacto de la migración de retorno en Tubuai, destacando doble propósito: por un lado, aislar los factores responsables de la migración de retorno y de este modo ubicar el fenómeno en su contexto histórico-estructural; y por el otro, valorar el impacto del retorno sobre la sociedad rural de origen (Tahití).

Por otro lado, Thomas-Hope (1999) argumenta que la migración de retorno a Jamaica (desde Estados Unidos, Inglaterra y Canadá) está estrechamente relacionada con la existencia y naturaleza de los vínculos transnacionales establecidos entre los migrantes y su país de origen, en especial a nivel de hogar y de familia.

Un trabajo por demás interesante es el realizado por Cohen y Haberfeld (2001) en Israel; con base en el método cuantitativo, analizan la autoselección de los migrantes retornados desde Estados Unidos que llegaron a este país entre 1970 y 1979, y regresaron a Israel entre 1980 y 1989. Su relevancia teórica estriba en hacer notar que, a pesar de existir tres modelos que analizan el retorno (el primero tiene que ver con un movimiento planeado, el segundo se ve como un fracaso debido a la mala información que se tenía y el tercero abarca la autoselección inmigrante que incluye la migración de retorno), ninguno se excluye mutuamente: es posible, dicen 
los autores, que el retorno de un país específico presente diferentes situaciones, y es necesario un entrecruzamiento de modelos.

Pero la investigación de Maron y Connell (2008), con base en un método cualitativo, analiza la migración de retorno en la villa de Nukunuku en Tonga, en las islas del Pacífico. Con una muestra de veinticinco emigrantes retornados, los autores concluyen que la migración ocurre principalmente por motivos de empleo y educación, mientras que el retorno es determinado por el contexto social y la responsabilidad del hogar (especialmente la necesidad de cuidar a los parientes mayores y abuelos, o sea que hay un motivo familiar fuerte para retornar).

Por su parte, Durand (2006) analiza la migración de retorno desde la ley de los rendimientos decrecientes que fue formulada originalmente por el economista francés Turgot (1727-1781), quien analizó los rendimientos no proporcionales, primero crecientes y luego decrecientes de la producción. En este punto, la toma de conciencia, por parte del migrante, de que se ha llegado a un límite, sea porque ha logrado el éxito o porque ya no es posible recibir mayores ingresos, lo coloca en una disyuntiva en la que tiene que evaluar nuevamente costos y beneficios económicos, sociales, familiares y personales (Durand, 2006), que constituyen los factores que impulsan el regreso.

En general, los modelos teóricos cualitativos y cuantitativos tienen conceptualizaciones muy diferentes en cuanto a la migración de retorno. Por ello, es difícil considerar a todos, por lo que enfatizamos que en este estudio la vertiente cuantitativa incluye el Modelo de Correspondencia Múltiple, el cual permite agrupar a los municipios en cuatro grupos. Tal agrupación se debe a las variables de contexto, las cuales sesgan el retorno. Es decir, se busca responder ¿qué pasa con el lugar de llegada? ¿En qué contexto llegan, trabajan, viven, se insertan y reinsertan? El argumento siguiente tiene pertinencia con este trabajo:

La relación movilidad-establecimiento, particularmente el efecto que el retorno de los migrantes internacionales podría tener en un contexto urbano, localizado en la Zona Metropolitana de la Ciudad de México. Esto es, poner a prueba la hipótesis de que en México, el efecto de la migración internacional (y particularmente de los retornados) "se diluye" en el contexto urbano, y sólo se refleja de manera nítida en las localidades rurales, a través de cambios evidentes en los paisajes locales y la dinámica comunitaria (Rivera Sánchez (2009). Esto parece ser congruente con las hipótesis planteadas en este trabajo: es decir, el migrante en su retorno tiene mayor presencia en los contextos metropolitanos y en menor medida en las zonas rurales del Estado 
de México, que es afin a lo que plantean Cohen y Haberfeld (2001) y Maron y Conelly (2008).

\section{Los ANTECedentes sobre la migración de Retorno en MéXICo}

Uno de los trabajos clásicos para entender la migración entre México y Estados Unidos es el de Massey y Espinosa (1997), quienes establecen que existen por lo menos cinco consideraciones fundamentales a la hora de decidir el retorno: i) el capital humano, conformado por las habilidades, conocimientos y capacidades adquiridas en el extranjero; ii) el capital social o redes sociales en ambas partes, por lo que consideran los autores que entre más familiares se establezcan en Estados Unidos, las posibilidades de retorno y contacto con la comunidad de origen disminuyen con el tiempo; iii) capital físico o material, o el número de propiedades, terrenos, parcelas agrícolas con las que cuenta en su comunidad de origen; iv) las condiciones económicas de la comunidad de origen, donde la diversificación económica y las posibilidades de inversión juegan un papel importante en la duración de la estancia en el extranjero; v) las condiciones macroeconómicas en ambos países, en donde la inflación, crisis y devaluación influyen a la hora de retornar e invertir. Esto nos permite comprender cómo este proceso migratorio está determinado por una tensión constante entre quedarse en Estados Unidos o retornar a México.

El retorno de los migrantes internacionales a sus lugares de origen constituye uno de los temas de mayor interés en el contexto de la crisis económica mundial reciente que inició en Estados Unidos en 2008. Muy poco se discute sobre la conceptualización de los retornos, sin embargo, Canales y Montiel (2007) destacan que los migrantes laborales de retorno son personas de 12 años o más que declararon haber ido a Estados Unidos a trabajar. Por su parte, Corona y Tuirán (1988) definen al migrante de retorno como aquella persona que cumple con dos condiciones: los sujetos de 12 años o más que fueron a Estados Unidos a vivir, trabajar y que consideraron ese viaje como un cambio de residencia. Pero que el concepto de "migración de retorno" refiere a movimientos en el espacio territorial de cierta población que regresa al punto de partida u origen. Pero esta mirada esconde varios supuestos: que las personas que retornan lo hacen a su localidad de origen, que se reinsertan en la dinámica local de donde habían partido y, finalmente, que este regreso significa a priori un retorno permanente (CONAPO, 2015c), dicho concepto es afin a la perspectiva demográfica, la cual es captada por la Encuesta Nacional de Dinámica Demográfica (ENA- 
DID) de 1992, 1997, 2006, 2009 y 2014, y la Encuesta Nacional Empleo (ENE) de 2002, y hoy se conoce como la Encuesta Nacional de Ocupación y Empleo (ENOE).

El análisis de la migración de retorno ha ocupado un lugar central dentro de los estudios migratorios en México, a raíz de la crisis económica de 2008. En este tenor, Canales (2012), sostiene que el retorno de mexicanos, aunque se incrementa, mantiene los niveles y tendencias que ya se visualizaban desde la década de los noventa. Por tanto, ni se ha dado un retorno masivo de millones de migrantes a sus comunidades y entidades de origen en México u otro lugar, como tampoco parece haberse dado un freno total de la emigración de mexicanos, que pudiera derivar, eventualmente, en la interrupción total del flujo migratorio. Este argumento coincide con algunos otros estudios como los de Rivera (2009); Dustmann et al. (1996), Ramírez y Aguado (2013) y Durand (2004), quienes se han apoyado en los supuestos de los enfoques teóricos vigentes de la migración internacional, argumentando que la decisión de regresar al país de origen es similar a la que se toma al momento de emigrar, es decir, se reinicia el proceso migratorio en sentido inverso y se ingresa nuevamente a una fase de toma de decisiones. Dichos autores, cuyas vertientes son cuantitativas y cualitativas, coinciden en señalar que en el retorno influyen diversos factores macro y microeconómicos, tales como las crisis económicas, las políticas migratorias, la dimensión sociodemográfica como la edad, sexo, el estado civil, la dimensión de capital humano, tiempo de estancia en Estados Unidos, dominio del idioma del país receptor, la escolaridad, el estatus migratorio y las redes sociales, entre otros.

Dichas vertientes han trabajado con dos argumentos: por un lado, los que coinciden en que la crisis económica de 2008 ha sido uno de los factores que influyeron en un mayor retorno desde Estados Unidos y se enfatiza en su falta de trabajo (Ramírez y Aguado, 2013), y por otro quienes sostienen que los retornos no son masivos por motivos laborales sino más bien por motivos familiares (Canales, 2012), pero muy poco se ha discutido sobre los factores o contexto de llegada.

Para ambos factores (el trabajo y motivos familiares) existe un antecedente histórico en México que da cuenta de ello. Para los motivos familiares y redes sociales encontramos el primer acontecimiento histórico marcado después del Tratado de Guadalupe Hidalgo (en inglés Treaty of Guadalupe Hidalgo) en 1848. El tratado estableció que México cedería más de la mitad de su territorio, que comprende la totalidad de lo que hoy son los estados de California, Nevada, Utah, Nuevo México y Texas, y partes 
de Arizona, Colorado, Wyoming, Kansas y Oklahoma. Por ello el gobierno de México publicó un decreto el 19 de agosto de 1848 en que ofrecía a todo aquel mexicano que lo solicitara trasladarse a territorio mexicano por cuenta del erario público y recibiría una dotación de tierras (Granados y Pizarro, 2013). Se calcula que cerca de 25 por ciento de la población de esos territorios regresó a México (Verduzco, 1995, citado en Granados y Pizarro, 2013).

Por el factor trabajo, tenemos que la crisis económica de 1929, fue el primer acontecimiento histórico al respecto, pues al aumentar el desempleo los gobiernos hicieron lo posible para reservar a sus connacionales los puestos de trabajo disponibles. Subsecuentemente, en este año, los mexicanos que habían sido bienvenidos se convirtieron en indeseables con el colapso de la economía y el inicio de la Gran Depresión. De 1929 a 1939, un grupo de 469 mil ciudadanos mexicanos fueron invitados o forzados a salir de Estados Unidos de América, muchos de ellos acompañados por sus hijos que eran ciudadanos americanos (Massey et al., 2009).

Por su parte, el INEGI (2016: 4) sostiene que:

...si se indaga el motivo de emigración en las personas que salen del país, también se obtiene información sobre el motivo de regreso a México. De acuerdo con los datos de la ENADID 2014, reunirse con la familia (45.7 por ciento), la falta de trabajo (20.5 por ciento) y la finalización de los estudios (11.7 por ciento) son las tres principales causas para que los emigrantes regresen al país. La reunificación familiar es el principal motivo tanto para los varones (45.5 por ciento) como para las mujeres (46.2 por ciento); sin embargo, al revisar la segunda y tercera causa son distintas. En los hombres, la falta de trabajo (23.9 por ciento) y la deportación (9.2 por ciento) completan el esquema; en las mujeres el término de estudios ( 21.5 por ciento) es más recurrente que la falta de empleo (10.8 por ciento).

Pero dicha información no recaba sobre la permanencia y el contexto de llegada. Con base en estos datos se plantea lo siguiente: el contexto urbano metropolitano y urbano de México orienta o le imprime cierta incertidumbre a la migración internacional de retorno y está ligado a la intensidad migratoria, la pobreza y la marginación, pero además condiciona los términos de su inserción y reinserción laboral, familiar y comunitaria. 
Factores contextuales de la migración de retorno a los municipios de las Zonas Metropolitanas ... / J.G. GONZÁLEZ y E.A. SANDOVAL

\section{INCORPORACIÓN PLENA DE LA MIGRACIÓN DE MEXIQUENSES}

\section{a Estados Unidos}

La migración mexiquense a Estados Unidos tiene su origen a principios del siglo XX (González, 2002). Desde entonces ha contribuido a los cambios en los patrones o modalidades de la migración circular o temporal hasta el proceso de asentamiento permanente de los migrantes mexiquenses en aquel país; además de la emigración, ${ }^{1}$ se ha presentado un aumento de la migración de retorno. Antes de analizar la migración de retorno, se mostrarán algunos indicadores básicos de incorporación plena de la migración a Estados Unidos (González y Montoya, 2012):

La emigración de los mexiquenses a Estados Unidos se ha posicionado a nivel nacional al pasar del vigésimo lugar en 1970, al cuarto, a partir del año 2000 y hasta 2014. En el año 2000 se estimaba que salían del Estado de México 99 mil migrantes al año; en el marco de la crisis de 2008, salieron del Estado de México 39 mil migrantes, aportando de esta manera a la migración permanente a Estados Unidos, cuyo estimado es de un millón doscientos mil hasta 2014.

En cuanto a la recepción de remesas monetarias que envían los migrantes desde el exterior a sus familiares, se reconoce que en 1970 la entidad ocupaba el lugar 18 y en 2015 el cuarto. Dichos ingresos, según Cervantes (2012), representan casi dos por ciento del Producto Interno Bruto (PIB) de la Entidad (1.6 por ciento). En 2007 las remesas familiares se estimaban en 2,172 millones de dólares y en 2015 su valor se ubicó en 1,561 millones de dólares. Pero lo que cabe destacar es que entre 2007 y 2015, el Estado de México dejó de recibir 610 millones de dólares porque muchos mexiquenses radicados en Estados Unidos perdieron su empleo y por ende dejaron de enviar dinero a sus familias. Esto ocurrió a causa de la crisis económica de 2008 surgida en Estados Unidos, que impactó en el mercado laboral de los migrantes, principalmente en la industria de la construcción en dicho país.

La definición operacional de la migración de retorno en las fuentes de información existentes en México difieren en cuanto a los propósitos en la medición y estimación, así como en el tamaño de muestra, naturaleza, institución y autor de que se trate. Por ejemplo, a través de las encuestas sociodemográficas, los censos y conteos de población y vivienda se pue-

\footnotetext{
1 Conapo define a la emigración como la acción mediante la cual una persona deja de residir en una unidad geográfica determinada (municipio o delegación, entidad federativa o país), para establecer su residencia habitual en otra, y la inmigración son quienes llegan a una unidad geográfica y en un periodo determinado. Véase en Glosario - CONAPO, disponible en www.conapo. gob.mx/es/CONAPO/Glosario_Migracion_Interna
} 
de estimar y trabajar con base en el retorno de los últimos cinco años, al preguntar a los encuestados por el lugar de residencia cinco años antes de su levantamiento. Por tanto, se estaría refiriendo al movimiento migratorio reciente. $\mathrm{O}$ en su defecto, quienes son entrevistados en distintos puertos fronterizos de México-Estados Unidos o aeropuertos y que son reconocidos como migrantes procedentes de Estados Unidos. Dichos registros se pueden encontrar en la Encuesta sobre Migración en la Frontera Norte de México (EMIF), publicada por el Colegio de la Frontera Norte. Estos son sin duda antecedentes muy importantes existentes en México para tratar el tema de la migración de retorno. Estas son algunas fuentes de información que se utilizan en este trabajo y destaca a personas que alguna vez fueron a trabajar o vivir a Estados Unidos en los últimos cinco años. Igualmente, se incluye a quienes nacieron en el vecino país del norte, para fines de la comparación y a quienes se reconoce como inmigrantes deportados u otra causa de su retorno.

Con base en las definiciones anteriores, se obtiene la participación de la migración de retorno de los mexiquenses desde Estados Unidos. Por ejemplo, las cifras del Instituto Nacional de Estadística y Geografía (INEGI) muestran un patrón bastante irregular que, sin embargo, permite afirmar que aumentó el tamaño de los retornos mexiquenses, de 40,550 personas entre 1987-1992 (esto supone en promedio 8,110 personas al año) a 45,615 entre 1992-1997 (en promedio 9,127 personas al año), con un mayor crecimiento en las zonas urbanas de la entidad (en términos absolutos el 80 por ciento de la población vive en zonas urbanas, por lo tanto va a haber más población migrante de retorno en términos absolutos en áreas urbanas...) (INEGI, 1992 y 1997). Después del año 2000, pero sobre todo en el periodo de crisis económica, los regresos hacia el Estado de México en 2009 reportaron una cifra que se ubicó en alrededor de 29 mil, hallazgos del Centro de Investigación y Estudios Avanzados de la Población (CIEAP) con base en la EMMEU, y sin embargo, con la EMIF dicha cifra se ubica hasta 42 mil migrantes de retorno (la diferencia obedece a que son dos metodologías distintas de la captura del regreso del migrante). Después de 2009, en 2014 y en años recientes el dato se ubicó en 17 mil (que residía en Estados Unidos u otro país cinco años antes al levantamiento de la información) (véase Tabla 1).

Para Blanco, (2000) el retorno de los migrantes a sus hogares significa fracaso o emerge como símbolo del éxito migratorio. 
Factores contexłuales de la migración de retorno a los municipios de las Zonas Metropolitanas ... / J.G. GONZÁLEZ y E.A. SANDOVAL

Tabla 1: Migración de retorno según fuente de información disponible

\begin{tabular}{|c|c|c|c|c|c|}
\hline Conceptos & Años & Nacional & $\begin{array}{l}\text { Estado de } \\
\text { México }\end{array}$ & Porcentaje & Fuente \\
\hline $\begin{array}{l}\text { Retorno durante los } \\
\text { últimos cinco años }\end{array}$ & $\begin{array}{l}1987 \\
1992\end{array}$ & 882,326 & 40,550 & 4.6 & ENADID, 1992 \\
\hline $\begin{array}{l}\text { Retorno durante los } \\
\text { últimos cinco años }\end{array}$ & $\begin{array}{l}1990 \\
1995\end{array}$ & 387,907 & 26,619 & 6.9 & $\begin{array}{l}\text { Conteo de } \\
\text { Población y } \\
\text { Vivienda, 1995: }\end{array}$ \\
\hline $\begin{array}{l}\text { Retorno durante los } \\
\text { últimos cinco años }\end{array}$ & $\begin{array}{l}1992 \\
1997\end{array}$ & 704,298 & 45,615 & 6.5 & ENADID, 1997 \\
\hline $\begin{array}{l}\text { Retorno durante los } \\
\text { últimos cinco años }\end{array}$ & $\begin{array}{l}1995 \\
2000\end{array}$ & 260,650 & 20,550 & 7.9 & Censo, 2000 \\
\hline $\begin{array}{l}\text { Retorno durante los } \\
\text { últimos cinco años }\end{array}$ & $\begin{array}{l}2000- \\
2005\end{array}$ & 244,244 & 12,563 & 5.1 & $\begin{array}{l}\text { Conteo de } \\
\text { Población y } \\
\text { Vivienda, } 2005\end{array}$ \\
\hline $\begin{array}{l}\text { Migrantes procedentes } \\
\text { de USA vía terrestre }\end{array}$ & 2005 & 816,907 & 21,780 & 2.7 & $\begin{array}{l}\text { COLEF, } \\
\text { CONAPO, } \\
\text { STPS, 2005 }\end{array}$ \\
\hline $\begin{array}{l}\text { Retorno durante los } \\
\text { últimos cinco años }\end{array}$ & $\begin{array}{l}2004 \\
2009\end{array}$ & 460,703 & 12,260 & 2.7 & ENADID, 2009 \\
\hline $\begin{array}{l}\text { Retorno durante los } \\
\text { últimos cinco años }\end{array}$ & $\begin{array}{l}2009 \\
2014\end{array}$ & 264,055 & 29,372 & 11.12 & ENADID, 2014 \\
\hline $\begin{array}{l}\text { Retorno durante los } \\
\text { últimos cinco años }\end{array}$ & $\begin{array}{l}2004 \\
2009\end{array}$ & - & 29,042 & - & CIEAP, 2009 \\
\hline $\begin{array}{l}\text { Retorno durante los } \\
\text { últimos cinco años }\end{array}$ & $\begin{array}{l}2005- \\
2010\end{array}$ & 350,719 & 30,946 & 8.8 & Censo, 2010 \\
\hline $\begin{array}{l}\text { Migrantes procedentes } \\
\text { de USA vía terrestre }\end{array}$ & 2009 & $1,196,981$ & 42,786 & 3.6 & $\begin{array}{l}\text { COLEF, } \\
\text { CONAPO, } \\
\text { STPS, } 2009\end{array}$ \\
\hline $\begin{array}{l}\text { Migrantes procedentes } \\
\text { de USA vía terrestre }\end{array}$ & 2011 & 799,986 & 22,397 & 2.8 & $\begin{array}{l}\text { COLEF, } \\
\text { CONAPO, } \\
\text { STPS, } 2011\end{array}$ \\
\hline $\begin{array}{l}\text { Retornos durante los } \\
\text { últimos cinco años }\end{array}$ & 2014 & 390,635 & 17,164 & 4.4 & ENADID, 2014 \\
\hline $\begin{array}{l}\text { Migrantes de retorno } \\
\text { durante los últimos cinco } \\
\text { años }\end{array}$ & 2015 & & 17,022 & & $\begin{array}{l}\text { INEGI, } \\
\text { Encuesta } \\
\text { Intercensal, } \\
2015\end{array}$ \\
\hline
\end{tabular}


Con esta breve cita de cifras se reconoce la incorporación plena de la migración mexiquense a Estados Unidos, independientemente de la fuente de información referida. Pero muy poco se sabe del contexto de su regreso y por tanto, presenta retos y desafíos para su atención. Uno de los principales retos de las instituciones académicas es hacer propuestas conceptuales y metodológicas para estimar de manera correcta la migración de retorno y los retos de la inserción, reinserción, según sea contexto urbano o rural. Pero el reto más importante continúa siendo la atención de política pública en los niveles nacionales, estatales y municipales.

\section{MODELO DE CORRESPONDENCIA MÚlTIPLE: ANÁLISIS DE RESULTADOS DE} LOS FACTORES CONTEXTUALES DE ORIENTACIÓN DE LA MIGRACIÓN DE Retorno al Estado de MéXico 2010-2015

El Análisis de Correspondencia Múltiple (ACM) es una técnica estadística que se utiliza para estudiar la relación entre variables categóricas que se exponen en una tabla de contingencia de los resultados de la migración de retorno (Greenacre, 1993; González et al., 2016). Esta técnica ayuda a explorar los datos y permite conocer los principales vínculos que se generen a partir de dicha tabla. Según Greenacre (1993) "la principal meta del análisis de correspondencia es transformar una tabla de información numérica en una de visualización gráfica”. En el presente trabajo, el ACM sirve para profundizar en la caracterización de la migración de retorno y el contexto de llegada del Estado de México, así como observar vínculos con los determinantes de la dimensión social. En la elaboración del ACM se usaron las siguientes herramientas básicas de esta técnica estadística: perfiles, masa, distancia, inercia y dimensiones.

"El ACM puede tener tantas dimensiones como $n-1$ categorías tenga la variable con mayor categoría" (Mancini, 2003). En esta investigación las variables con más categorías son el Índice de Marginación, el Índice de Intensidad Migratoria y el municipio, los cuales cuentan con categorías y dimensiones posibles de relación para éste estudio. Para proceder a correr el modelo se tuvieron que transformar las variables numéricas en categóricas o cualitativas tal como se expone en la Tabla 2.

La Tabla 2 muestra cómo se construyó la base de datos para 2010-2015 con variables cualitativas para luego correrla en Statistical Package for the Social Sciences, 21, (por sus siglas en inglés SPSS 21) en etapas, las cuales ayudan a comprender de mejor manera esta técnica estadística. El ACM permite construir una tabla de contingencia de dos variables, en la que se puede verificar una primera relación entre éstas. Con base en ello se pue- 
den construir tablas de frecuencia de doble entrada y la representación gráfica de la tabla de contingencia, que es uno de los atributos de esta técnica estadística, la cual exhibe con mayor claridad los vínculos existentes entre las variables de estudio. Este trabajo consideró cinco variables incluidas en la Tabla 2, mostrando las categorías que se reflejan en la gráfica de esta técnica estadística que a continuación se expone.

Tabla 2: Proceso de codificación por correspondencia sobre la migración de retorno y sus determinantes. Migración de retorno 2010-2015

\begin{tabular}{|c|c|}
\hline Indicador a nivel municipal & Categorías \\
\hline & $(0-0.600=$ Bajo $)(0.601-0.997=$ Medio $)$ \\
\hline Retorno (reto & $(0.997-1.2=$ Medio alto $)(1.2-12=$ Alto $)$ \\
\hline Índi & $(-0.8100$ thru $-0.3200=$ Muy bajo $)(-0.3300$ thru \\
\hline migrator & $0=$ Bajo $)(0.01$ thru $1.90=$ Medio $)(0.9300$ thru - \\
\hline$(2015))$ & $0.8000=$ Alto $)$ \\
\hline Índice de marginación & $(-2$ thru $-0.80=$ Muy alto $)(-0.79$ thru $-0.50=$ \\
\hline IM2010 & $\begin{array}{l}\text { Alto }) \\
(-.51 \text { thru }-0.2=\text { Medio })(-0.2 \text { thru } 0.9=\text { Bajo })\end{array}$ \\
\hline & u $49=$ Medio $)$ \\
\hline PPE & $(74$ thru $90=$ Muy alto $)$ \\
\hline $\begin{array}{l}\text { Zona metropolitana } \\
\text { (V/y ZM) }\end{array}$ & $\begin{array}{l}\text { Zona Metropolitana de la Ciudad de México } \\
\text { (1);Toluca (2);Tianguistenco (3) y Municipios } \\
\text { no metropolitanos (4) }\end{array}$ \\
\hline
\end{tabular}

Fuente: INEGI, 2010; INEGI, 2015; CONAPO, 2015a; CONAPO, 2015b; CONEVAL, s/f; CONAPO, 2012; INEGI, 2015.

\section{ANÁlisis de RESUltados del CONTEXTO DE LA MIGRACIÓN DE RETORNO}

El Análisis de Correspondencias Múltiple (ACM) es una técnica descriptiva para representar tablas de contingencia. Los datos de partida para el análisis de correspondencias es una matriz $X$ de dimensiones $n \times k$ que representa las frecuencias absolutas observadas en una tabla de contingencia de dos variables, donde la primera se representa por filas y tiene $\mathrm{n}$ categorías y la segunda por columnas y tiene $k$ categorías. Por ejemplo, se clasifica un conjunto de municipios en $n$ posibles tasas de rechazo al retorno y $k$ posibles tasas de atracción en el retorno. En general, el elemento $X_{\mathrm{ij}}$ de la matriz $X$ representa la frecuencia absoluta observada en la casilla $(i, j)$ de la tabla de contingencia. En esencia, es un tipo especial de análisis de componentes principales pero realizado sobre una tabla de contingencia y usando una distancia euclídea ponderada llamada chi-cuadrado. Esta metodología 
se usa para analizar datos de encuestas y censos de población y vivienda, ya que permite mirar similitudes y cercanías de las variables. Para ello se transformaron las variables cuantitativas en cualitativas o nominales.

Se tomó como referencia este modelo porque es el que más se ajusta para evaluar los determinantes de la migración de retorno y que a la vez permite ver sus determinantes socioeconómicos para incentivar o desincentivar la llegada desde Estados Unidos, específicamente, con esto es posible evaluar con este modelo la proximidad entre municipios en términos de parecido en su atracción o rechazo: dos municipios se parecen si tienen casi las mismas modalidades en sus determinantes socioeconómicos como la pobreza, la marginación o la intensidad de la migración de retorno.

El modelo permite, por otra parte, verificar la proximidad entre modalidades de variables diferentes en términos de asociación en el retorno (o están en el mismo cuadrante): si son cercanos puesto que globalmente están presentes en los mismos municipios que es coincidente con su intensidad migratoria u otra variable de contexto incluida.

El modelo permite verificar la proximidad entre modalidades de una misma variable en términos de su parecido: son excluyentes por construcción, si son cercanas o están en el mismo cuadrante es porque los municipios que las poseen presentan casi el mismo comportamiento en las otras variables determinantes socioeconómicas.

Con base en un ejercicio básico, se puede decir que el retorno a municipios urbanos y no urbanos en los 125 municipios del Estado de México se resume en las tablas y figuras siguientes.

En la Tabla 3 se resumen los modelos en donde se aprecian las dimensiones de la ecuación que expresan la magnitud de la varianza que por un lado, la dimensión 1 aporta 80 por ciento y el segundo 34 por ciento de los cambios que hay en la migración de retorno para 2010 y, en 2015 estas cifras se ubicaron en 70 y 32 por ciento. Pero ¿qué variables quedaron en la dimensión uno y dos? ¿Qué tanto aportan las variables? La varianza indica que es alto, lo cual muestra que es explicado por estas dos dimensiones y las magnitudes tienen igual importancia, ${ }^{2}$ lo cual habla de que es un buen modelo para explicar la orientación de retorno. Los autovalores e inercias indican el mayor peso de la primera dimensión por sobre la segunda y debe ser tomado en cuenta para interpretar $\mathrm{y}$, por consiguiente permitirá interpretar la Figura 1 que muestra las cinco variables y que se presenta más adelante (De Angelis, 2011).

\footnotetext{
2 El uno está agrupando a los municipios urbanos y los dos municipios rurales.
} 
Factores contextuales de la migración de retorno a los municipios de las Zonas Metropolitanas ... / J.G. GONZÁLEZ y E.A. SANDOVAL

Tabla 3: Estadísticos

\begin{tabular}{lcccc}
\hline \multicolumn{5}{l}{ Resumen del modelo 2010} \\
\hline Dimensión & $\begin{array}{c}\text { Alfa de } \\
\text { Cronbach }^{\text {a }}\end{array}$ & $\begin{array}{c}\text { Total } \\
\text { (Autovalores) }\end{array}$ & Inercia & $\begin{array}{c}\text { \% de la } \\
\text { varianza }\end{array}$ \\
\hline 1 & 0.938 & 4.004 & 0.801 & 80.075 \\
2 & 0.534 & 1.746 & 0.349 & 34.91 \\
Total & & 5.749 & 1.15 & \\
Media & $0.815 \mathrm{a}$ & 2.875 & 0.575 & 57.493 \\
\hline
\end{tabular}

Resumen del modelo 2015

\begin{tabular}{|c|c|c|c|c|}
\hline \multicolumn{5}{|c|}{ Varianza explicada } \\
\hline Dimensión & $\begin{array}{c}\text { Alfa de } \\
\text { Cronbach }^{\mathrm{a}}\end{array}$ & $\begin{array}{c}\text { Total } \\
\text { (Autovalores) }\end{array}$ & Inercia & $\begin{array}{c}\% \text { de la } \\
\text { varianza }\end{array}$ \\
\hline 1 & 0.859 & 3.2 & 0.64 & 70.075 \\
\hline 2 & 0.465 & 1.592 & 0.318 & 32.91 \\
\hline Total & & 4.793 & 0.959 & \\
\hline Media & $0.728 \mathrm{a}$ & 2.396 & 0.479 & 56.49 \\
\hline
\end{tabular}

Teniendo presente que la dimensión 1 representa a municipios con mayor propensión al de retorno en 2015 y en menor medida en 2010 (cuadrante I: retorno bajo), dimensión 1, el cuadrante II le siguen los municipios rurales de mayor propensión al retorno (retorno medio), dimensión 2, el cuadrante III, retorno medio alto, dimensión 2 y el IV el retorno alto, dimensión I (Tabla 1 y Figura 2) con base en la tabla de discriminación se puede anotar lo siguiente:

La medida discriminante indica que las variables que más aportan al sesgo en el destino de los retornados son la zona metropolitana (0.873), el índice de marginación (0.83), el índice de intensidad migratoria (0.82), el porcentaje de pobreza $(0.755)$ en 2010 , con pequeñas variantes para 2015 , con una reducción en las medidas de discriminación para 2015 en la dimensión 1. En términos generales se puede decir que los determinantes de mayor peso en la orientación de la migración de retorno municipal son la pertenencia a zonas metropolitanas, el índice de marginación, el índice de intensidad en el retorno lo cual se sugiere poner atención para definir la política pública estatal (Tabla 4). 
Figura 1: Medida de discriminación de 2010

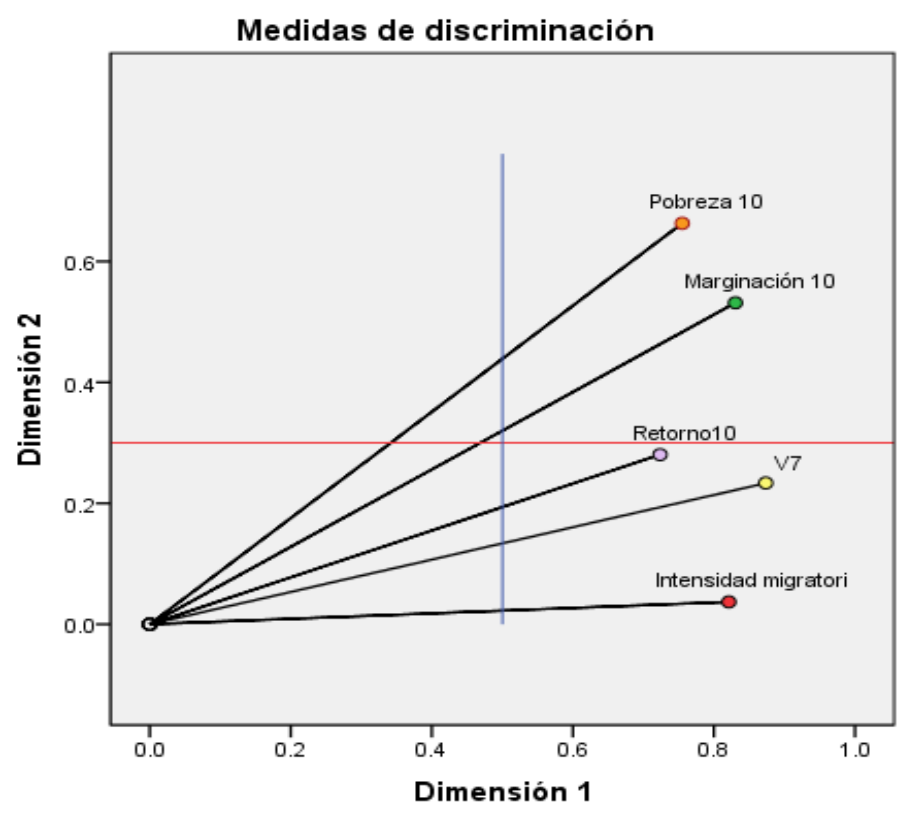

Normalización principal por variable.

Fuente: INEGI, 2010; CONAPO, 2015a, CONAPO, 2015b; CONEVAL, s/f, CONAPO, 2012.

Lo anterior se puede apreciar en las figuras 1 y 2 en sus dimensiones 1 y 2. Se espera que las medidas de discriminación "discriminen" las variables para cada factor o dimensión. Al respecto, en las dos dimensiones que elabora el modelo para 2010 y 2015, en la primera dimensión o eje se destaca la intensidad migratoria, la zona metropolitana y el retorno. En el segundo eje tiene preponderancia la pobreza y la marginación tanto en 2010 y 2015. En términos gráficos queda como lo indican las figuras 1 y 2 .

En las figuras 3 y 4 se puede apreciar la agrupación de los municipios en los cuatro cuadrantes o grupos según condición de recepción de migrantes de retorno, las variables socioeconómicas que son causales de su sesgo en la llegada desde el exterior. Los grupos se dividen de la siguiente manera:

En el primer cuadrante tenemos a un grupo de 21 municipios en 2010 y en 2015 a 23, en él se encuentran los municipios rurales en situación de recepción de migrantes de retorno (estos llegan principalmente a las cabeceras municipales), por ejemplo, el municipio número 87, que es Temascalcingo y se ubica del lado derecho, dimensión 2 y según los indicadores 
de pobreza, marginación, es un municipio vulnerable con un proceso de retorno muy bajo. Le siguen en orden de importancia Texcaltitlán (99), Amatepec (107), Otzoloapan (68), Ixtapan del Oro (43), San José del Rincón (76), Sultepec (82), Coatepec Harinas (28), Acambay (1), Donato Guerra (33), Ocuilán (66), San Simón de Guerrero (79), Malinalco (55), Almoloya de Alquisiras (4), Aculco (3) y Tonatico, entre otros. Algunos municipios citados pertenecen a un grupo con rechazo alto y muy alto de la migración de retorno entre el periodo 2010 y 2015. Destacan municipios ligados a la alta marginación, pobreza y sobre todo a la migración internacional, principalmente hay más de 25 en el Sur del Estado de México. Dichos municipios son de zonas rurales reconocidas como lugar de alta tradición migratoria y de reciente incorporación como los municipios del noroeste de la entidad mexiquense (González, 2012).

Figura 2: Medida de discriminación de 2015

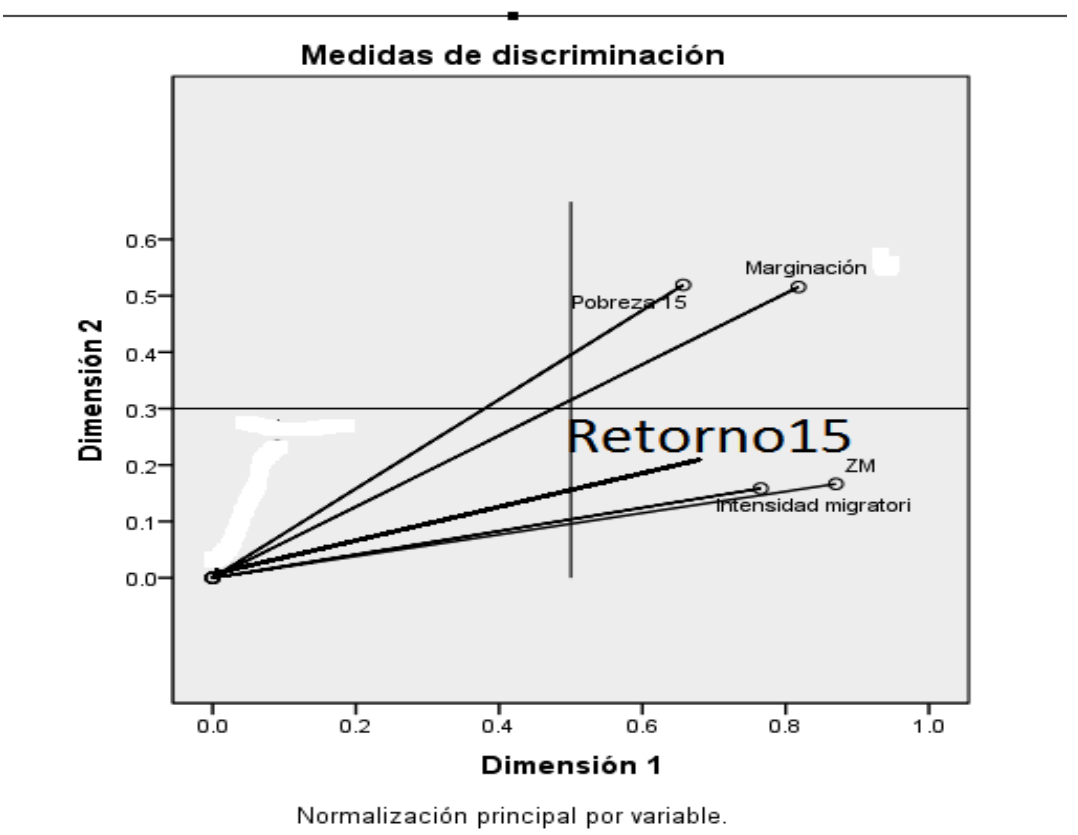

Fuente: INEGI, 2010; CONAPO, 2015a, CONAPO, 2015b; CONEVAL, s/f, CONAPO, 2012. 
Tabla 4: Medidas de discriminación 2010 y 2015

\begin{tabular}{|c|c|c|c|}
\hline \multicolumn{4}{|c|}{ Medidas de discriminación 2010} \\
\hline & \multicolumn{2}{|c|}{ Dimensión } & \multirow{2}{*}{ Media } \\
\hline & 1 & 2 & \\
\hline Retorno10 & 0.724 & 0.280 & 0.502 \\
\hline Intensidad migratoria & 0.821 & 0.037 & 0.429 \\
\hline Pobreza 10 & 0.755 & 0.663 & 0.709 \\
\hline Marginación 10 & 0.830 & 0.532 & 0.681 \\
\hline V7 & 0.873 & 0.234 & 0.554 \\
\hline Total activo & 4.004 & 1.746 & 2.875 \\
\hline \multicolumn{4}{|c|}{ Medidas de discriminación 2015} \\
\hline & \multicolumn{2}{|c|}{ Dimensión } & \\
\hline & 1 & 2 & Media \\
\hline Retorno10 & 0.800 & 0.233 & 0.516 \\
\hline Intensidad migratoria & 0.765 & 0.158 & 0.462 \\
\hline Pobreza 15 & 0.657 & 0.519 & 0.588 \\
\hline Marginación 10 & 0.818 & 0.516 & 0.667 \\
\hline $\mathrm{ZM}$ & 0.870 & 0.166 & 0.518 \\
\hline Total activo & 3.200 & 1.592 & 2.396 \\
\hline
\end{tabular}

Fuente: INEGI, 2010; CONAPO, 2015a; CONAPO, 201b5; CONEVAL, s/f; CONAPO, 2012.

Por ejemplo, Acambay y Temascalcingo, pertenecen a la región indígena mazahua-otomí y esto es un indicador de que dichos grupos indígenas se han incorporado a la migración internacional. La combinación de dispersión de la población, con infraestructura débil y mayor pobreza-marginación influyen en el ritmo y sesgo del retorno a sus comunidades de origen y en el medio rural (CEPAL, 2012; González et al, 2016).

En el cuadrante 2, también en la dimensión 2, se encuentran 34 municipios rurales en 2010 y 24 en 2015 , en términos numéricos disminuyó el número de municipios con recepción de migrantes de retorno entre estos dos puntos en el tiempo. Dichos municipios son Toluca (108), Zumpango (125), Metepec (57), Atizapán de Zaragoza (13), Huixquilucan (39), Cuautitlán Izcalli (32), Chicoloapan (24), Calimaya (18), Chapultepec (22), Nicolás Romero (63), Tecámac (83), Chalco (20), Cocotitlán (29), Tlalmanalco (105), Ayapango (17), Chiautla (23), Mexicalcingo (58), Huehuetoca entre otros. 
Figura 3: Centroides y objetos del diagrama de dispersión biespacial 2010

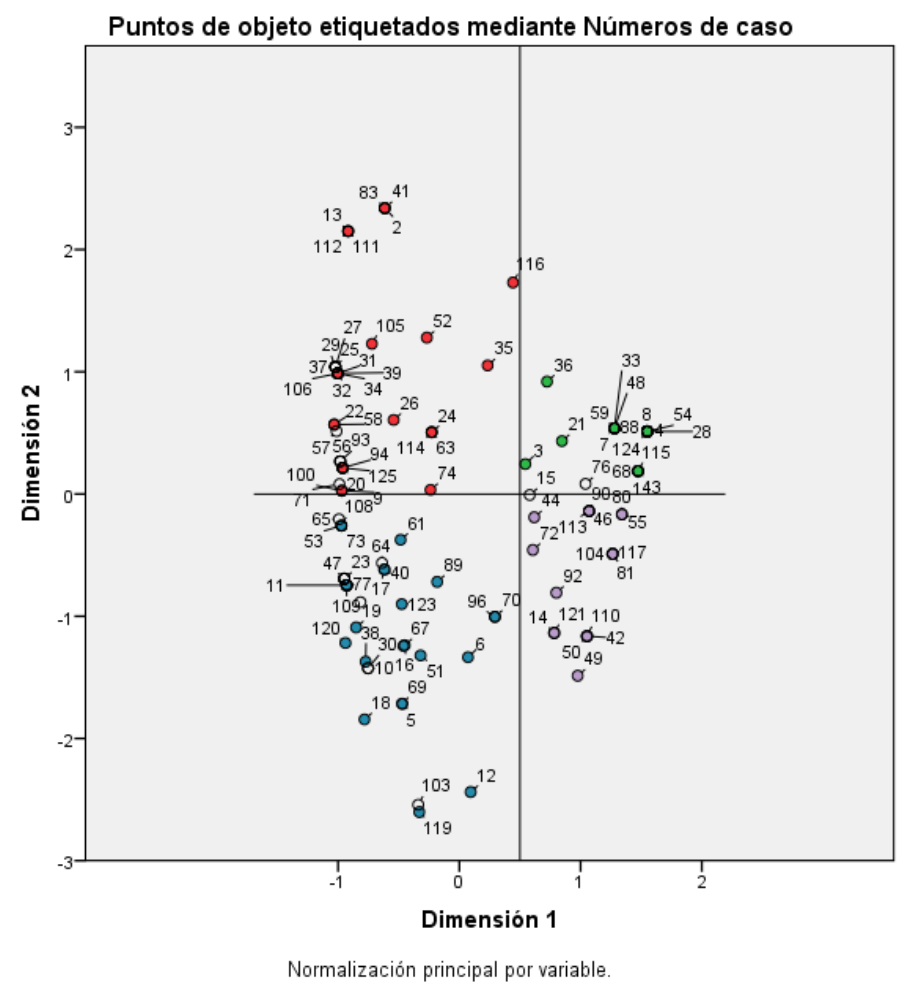

Fuente: INEGI, 2010; CONAPO, 2015a, CONAPO, 2015b; CONEVAL, s/f, CONAPO, 2012.

El ritmo del crecimiento de la atracción de estos municipios se explica en mayor parte por las condiciones económicas cada vez más favorables cuya ubicación geográfica es la Zona Metropolitana de la Ciudad de México, Toluca y Santiago Tianguistenco. ${ }^{3}$

Cabe destacar que los municipios localizados en la Zona Metropolitana de Toluca y Santiago Tianguistenco, muchos de ellos serán municipios dormitorios ${ }^{4}$ a raíz de la implementación del tren interurbano Ciudad de

3 Para verificar las delimitaciones Metropolitanas véase INEGI, Delimitación de las zonas metropolitanas de México - Inegi, disponible en www.inegi.gob.mx/est/contenidos/espanol/metodologias/otras/ZONAS_MET.pdf

4 Ciudad dormitorio: $\bar{B}$ arrios o municipios cercanos a las grandes ciudades. Sus habitantes se desplazan diariamente al trabajo y vuelven a la localidad a dormir (desplazamientos pendulares), véase en Historia y Geografía, disponible en sosiales.blogspot.com/2009/02/vocabulario-geografico-v-la-ciudad.html 
México-Toluca ${ }^{5}$ (González et al., 2016), y es donde existe mayor posibilidad de retorno. México-Toluca ${ }^{6}$ (González et al., 2016), y es donde existe mayor posibilidad de retorno.

Figura 4: Centroides y objetos del diagrama de dispersión biespacial 2015

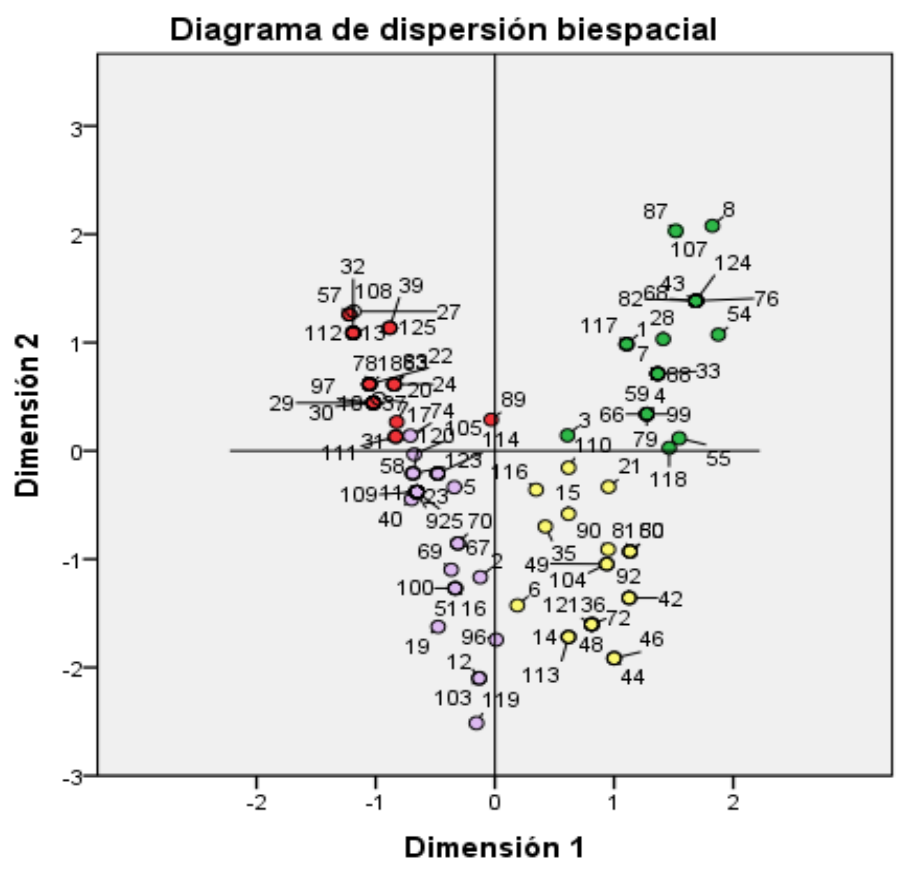

Normalización principal por variable.

Fuente: INEGI, 2010; CONAPO, 2015a; CONAPO, 2015b; CONEVAL, s/f, CONAPO, 2012.

Un tercer grupo de atracción de inmigrantes de retorno internacional se encuentra expresado en el cuadrante III, dimensión 1 y está representado por 29 municipios en 2010 y 21 municipios del Estado de México (véanse figuras 3 y 4). Aquí destacan los municipios que presentan un incipiente potencial en la atracción económica y poblacional. En este grupo se aglutinan los municipios de Cuautitlán (31), San Antonio la Isla (74), Coyotepec (30), Almoloya de Juárez (5), Atenco (11) Apaxco (10), Isidro Fabela (40), Amecameca (9), Otzolotepec (69), Ozumba (70), Otumba (67), Texcalya-

5 Para mayor información el proyecto del tren interurbano, véase en Tren Interurbano México-Toluca - Secretaría de Comunicaciones y Transporte, disponible en www.sct.gob.mx/transporte-y-medicina.y./tren-interurbano-mexico-toluca/

Para mayor información el proyecto del tren interurbano, véase en Tren Interurbano México-Toluca - Secretaría de Comunicaciones y Transporte, disponible en www.sct.gob.mx/transporte-y-medicina.y./tren-interurbano-mexico-toluca/ 
cac (100), Juchitepec (51), Axapusco (16), Acolman (2), Capulhuac (19), Atizapán (12), Tepetlixpa (96), Tianguistenco (103) y Xalatlaco (119) entre otros. Algunos de estos municipios están incorporándose paulatinamente a las principales metrópolis de la Ciudad de México y a la de Toluca, o no están muy alejados de los principales centro de desarrollo pero que son ejes o centros regionales económicos y administrativos (González et al., 2016).

En el último grupo de municipios tenemos a los ubicados en el cuadrante IV y dimensión 1, (véanse figuras 3 y 4), cuyas potencialidades económicas son incipientes y con un poder de atracción de la población también incipiente, los cuales son (González et al., 2016): Chiconcuac, Chapa de Mota, Villa del Carbón, Atlautla, Ecatzingo, Villa Victoria, Tenancingo, Soyaniquilpan de Juárez, Timilpan, Joquicingo, Jocotitlán, Tenango del Valle, Almoloya del Río, Ixtapan de la Sal, Atlacomulco, Atizapán de Zaragoza, Jiquipilco, Valle de Bravo, Ixtapaluca, Jilotepec, entre otros. Algunos de estos municipios se están incorporando paulatinamente a las principales metrópolis o están más alejados de los principales centro de desarrollo pero que algunos son ejes o centros regionales económicos y administrativos.

En síntesis, podemos decir que el modelo de correspondencia agrupó a los 125 municipios del Estado de México en cuatro grupos para 2010 y 2015. Uno con retorno rural y desconectado de los centros de desarrollo de la entidad y del país, el segundo con atracción urbana, el tercero con atracción ascendente con cercanía a las metrópolis y el cuarto con atracción a ciudades medias o centro regionales alejados de las ciudades centrales (Toluca y Ciudad de México). El grupo de mayor retorno son municipios Metropolitanos en donde los inmigrantes buscan un estilo de vida y trabajo similar a la de Estados Unidos, tanto para ellos como para los de su familia. Por ejemplo, el retornar a Nezahualcóyotl, Tlalnepantla, Naucalpan, Coacalco o Ecatepec, les permite moverse a la ciudad central (Ciudad de México) a ciudades cercanas o al interior del país.

Para el caso del retorno a los municipios rurales los pondría en desventaja en cuanto al trabajo, con alta posibilidad de desempleo y una baja en su nivel de vida sobre todo que en dichas zonas han estado en crisis económica permanente. A esto se agregan los conflictos familiares y el no contar con una vivienda propia, por lo que la alternativa es buscar tener una residencia en las principales Zonas Metropolitanas del país o ciudades medias donde contribuyen con sus experiencias, conocimientos y recursos al desarrollo de las zonas de destino en detrimento de las áreas de las cua- 
les la gente emigra, ya que en definitiva serían los que habrían de producir riqueza en su espacio rural (CEPAL, 2012).

Por el lado de las zonas metropolitanas, hay una mayor opción de trabajo, destacando en aquellos de incipiente y mediana competitividad económica sectorial, sobre todo, los municipios que pertenecen a la Zona Metropolitana del Valle de México y a las zonas metropolitanas de Toluca y Tianguistenco. En dichos municipios se avizora un cambio radical en su tamaño de población y su infraestructura, sobre todo, en cuanto empiece a funcionar el tren suburbano México-Toluca. Dicho medio de transporte por sí solo podrá generar nueva infraestructura y una mayor competitividad de algunos sectores económicos de las Zonas Metropolitanas de Toluca y Tianguistenco, porque las inversiones que apuntarán hacia la capital, ocasionarán que Toluca y Tianguistenco a la vez experimenten el fenómeno mayor de la inmigración, situación que ya vivieron demarcaciones como Nezahualcóyotl y Ecatepec al convertirse en "ciudad dormitorio" (González et al., 2016).

\section{Conclusiones}

Con base en los objetivos se concluye lo siguiente: la migración de retorno al Estado de México, principalmente desde Estados Unidos entre 2010 y 2015 presentó cambios importante en el lustro de análisis. Para algunos cuadrantes aumentó y en otros disminuyó como consecuencia propia de la dinámica de este fenómeno. La intensidad migratoria juega un papel fundamental en la orientación de la migración de retorno hacia municipios metropolitanos, y en menor medida hacia municipios rurales cuya determinación son principalmente por los niveles de la pobreza y la marginación. Sobre esto habrá que seguir trabajando en su nivel teórico y empírico para consensar o en su defecto contra-argumentar la influencia del contexto socioeconómico en la migración de retorno.

El uso del modelo de correspondencia múltiple permitió probar que en los contextos de mayor pobreza y de alta marginación sucede un menor retorno representados por los municipios rurales en el cuadrante 1, no así en las zonas urbanas, cuyas condiciones son muy parecidas a las que previamente ha vivido y trabajado el migrante internacional.

En términos generales, se sostiene que las variables de la pobreza, la marginación, la ubicación geográfica del municipio (metropolitanos vs rurales), así como la intensidad migratoria son factores que incide en la migración de retorno. Con base en esto podemos decir que llegamos a las siguientes conclusiones: tenemos municipios de zonas rurales que son 
reconocidos como lugar de alta tradición migratoria (los del sur del Estado de México) y de reciente incorporación a la migración internacional como los municipios del noroeste de la entidad mexiquense que pertenecen a la región indígena mazahua y otomí, los cuales presentan una débil atracción de dichos migrantes por su escasa infraestructura, mayor pobreza y marginación que son factores de rechazo del migrante para sentar su residencia.

Otros municipios, cuyo ritmo del crecimiento económico se vuelve atractivo para inmigrantes internos, pero sobre todos internacionales por las condiciones económicas cada vez más favorables, son los que conforman la Zona Metropolitana de la Ciudad de México, Toluca y Santiago Tianguistenco. Cabe destacar que algunos municipios de dichas zonas metropolitanas serán dormitorios a raíz de la puesta en marcha del tren interurbano Ciudad de México-Toluca.

Los municipios que se están incorporando paulatinamente a las principales metrópolis de la Ciudad de México y a la de Toluca o no están más alejados de los principales centro de desarrollo pero que son ejes o centros regionales económicos y administrativos, son atractores de los inmigrantes internacionales de retorno, con preferencia en las cabeceras municipales.

\section{REFERENCIAS BIBLIOGRÁFICAS}

Appleyard, R. T., 1962, "The return movement of United Yingdom migrants from Australia", en Population Studies, 15(3), 214-225. Disponible en www.tandfonline.com/

Blanco, Cristina, 2000, Las migraciones contemporáneas, Alianza Editorial.

Canales, Alejandro y Montiel, Israel, 2007, "De la migración interna a la internacional. En búsqueda del eslabón perdido". Ponencia presentada en el Taller nacional sobre migración interna y desarrollo en México: diagnóstico, perspectivas y políticas, México, Centro Latinoamericano y Caribeño de Demografía, 16 de abril.

Canales, A. I., 2012, “La migración mexicana frente a la crisis económica actual. Crónica de un retorno moderado", en Rev. Inter. Mob. Hum., Brasilia, 20(39), $117-$ 134, jul./dez, disponible en http://www.scielo.br/

CEPAL (Comisión Económica para América Latina y el Caribe), 2012, Población, territorio y desarrollo sostenible. Síntesis (LC/L.3475(CEP.2/4)), Santiago, mayo.

Cervantes, J., 2012, Comportamiento reciente del ingreso de México por remesas familiares. Disponible en http://www.cemla.org/

Cohen, Y. y Haberfeld, Y., 2001, "Self-selection and return migration: Israeli-Born jews returning home from the United States during the 1980s", en Population Studies, 55(1), 79-91, disponible en http://www.tandfonline.com/ 
COLEF, CONAPO, STPS, varios años, Encuesta sobre Migración en la Frontera Norte de México, disponible en https://www.colef.mx/emif/

CONAPO, 2012, Índice de marginación por entidad federativa y municipio 2010. Disponible en http://www.conapo.gob.mx/

CONAPO, 2015a, Indicadores sobre migración a Estados Unidos, índice y grado de intensidad migratoria por entidad federativa, 2015, disponible en www.conapo.gob.mx

CONAPO, 2015b, Índice de Marginación, 2015; Consejo Nacional de Población, México.

CONAPO, 2015c, El retorno en el nuevo escenario de la migración entre México y Estados Unidos, disponible en https:/www.gob.mx/cms/uploads/attachment/ file/39174/ElRetornoEnelNuevoEscenariodeMigracion.pdf

CONEVAL, s/f, Evolución de la pobreza por ingresos, disponible en http://www. coneval.gob.mx/

CONEVAL, s/f, Pobreza a nivel municipio 2010 y 2015. Disponible en https:// www.coneval.org.mx/

Corona Vázquez, R. y Tuirán, R., 1988, “Migración y retorno y migraciones sucesivas", en López Castro, G., Migración en el occidente de México, El Colegio de Michoacán, Zamora, disponible en http://books.google.com.mx/

Chapman, M. y Mansell Prothero, R., 1983, Themes on circulation in the Third World. Disponible en https://books.google.com.mx/

De Angelis, Carlos F., 2011, "Empleo del análisis de correspondencias múltiples para la identificación del acceso diferencial al sistema de medios de comunicación en la ciudad de Buenos Aires y el conurbano bonaerense 2007 - 2009", en $I X$ Jornadas de Sociología. Facultad de Ciencias Sociales, Universidad de Buenos Aires, Buenos Aires, 2011, disponible en http://cdsa.aacademica.org/000-034/644. pdf (consultado el 2/03/2017).

Durand, Jorge, 2004, "Ensayo teórico sobre la emigración de retorno. El principio del rendimiento decreciente", en Cuadernos Geográficos, (035), 103-116.

Durand, Jorge, 2006, “Los Inmigrantes también emigran”, en REMHU - Revista Interdisciplinar da Mobilidade Humana: año 14, - núm. 26 e 27.

Dustmann, C., Bentolila, S. y Faini, R., 1996, "Return migration: the European experience", en Economic Policy, 11(22), 213-250, disponible en www.ucl.ac.uk

Fernández Guzmán, E., 2011, "Revisión bibliográfica sobre la migración de retorno", en Norteamérica, 6(1), México enero/junio, disponible en http://www.scielo. org. $\mathrm{mx} /$

Glaser, W. A. y Habers, G. C., 1974, "The migration and return of professionals", en International Migration Review, 8(2), 227-244.

Goldstein, S., 1964, "The Extent of Repeated Migration: An Analysis Based on the Danish Population Register", en Journal of the American Statistical Association, 
Factores contextuales de la migración de retorno a los municipios de las Zonas Metropolitanas ... / J.G. GONZÁLEZ y E.A. SANDOVAL

59(308), 1121-1132. Disponible en http://www.tandfonline.com/doi/abs/10.1080/ 01621459.1964.10480753

Goldstein, S., 1964, "The extent of repeated migration: an analysis based on the danish population register", en Journal of the American Statistical Association, 59(308), 1121-1132. Disponible en http://www.tandfonline.com/

González Becerril, Juan Gabino, 2002, Migración laboral internacional del Estado de México, CIEAP-UAEMÉX. Disponible en https://books.google.com

González Becerril, J. G. y Montoya Arce, B. J. (compiladores), 2012, Migración mexiquense a Estados Unidos: un análisis interdisciplinario. Toluca: CIEAP-UAEM.

González Becerril, Juan Gabino, Montoya Arce, Bernardino Jaciel y Sandoval Forero, Eduardo Andrés, 2016, "Poblamiento y despoblamiento: dos caras de la desigualdad sociodemográfica en el estado de México", en Notas de Población núm. 103 julio-diciembre págs. 149-167. Disponible en https://repositorio.cepal. org/ (consultado el 05/01/2019).

Granados, José Aurelio y Pizarro Hernández, Karina, 2013, "Paso del Norte, qué lejos te vas quedando. Implicaciones de la migración de retorno en México", en Estudios Demográficos y Urbanos, vol. 28, núm. 2, El Colegio de México, México. pp. 469-496.

Greenacre, Michael J., 1993, Correspondence Analysis in Practice, Londres, Academic Press.

INEGI, 1992, Encuesta Nacional de la Dinámica Demográfica (ENADID), 1992, Instituto Nacional de Estadística y Geografía, México.

INEGI, 1995, Conteo de Población y Vivienda, 1995, Instituto Nacional de Estadística y Geografía, México.

INEGI, 1997, Encuesta Nacional de la Dinámica Demográfica (ENADID), 1997, Instituto Nacional de Estadística y Geografía, México.

INEGI, 2000, Censo General de Población y Vivienda de 2000, Instituto Nacional de Estadística y Geografía, México.

INEGI, 2005, Conteo de Población y Vivienda de 2005, Instituto Nacional de Estadística y Geografía, México.

INEGI, 2009, Encuesta Nacional de la Dinámica Demográfica (ENADID), 2009, Instituto Nacional de Estadística y Geografía, México.

INEGI, 2010, Censo de Población y Vivienda de 2010, Instituto Nacional de Estadística y Geografía, México.

INEGI, 2014, Encuesta Nacional de la Dinámica Demográfica (ENADID), 2014, Instituto Nacional de Estadística y Geografía, México.

INEGI, 2015, Encuesta Intercensal, 2015, Instituto Nacional de Estadística y Geografía, México. 
INEGI, s/f, Encuesta Nacional de Empleo (ENE), disponible en https://inegi.org. $\mathrm{mx} /$ contenidos/programas/migracion/2002/doc/cuest_migra02.pdf

Lee, A. S., 1974, "Return migration in the United States", en International Migration Review, 8(2), 283-300. Disponible en http://repository.forcedmigration.org/

Loockwood, V. S., 1990, "Development and return migration to rural French Polynesia", en International Migration Review, 24(2), 347-371. Disponible en disponible en http://thirdworld.nl/development-and-return-migration-to-rural-french-polynesia

Mancini, F., 2003, Trabajo y certidumbre: condiciones y percepciones de la inseguridad laboral en México, tesis de maestría en población, Ciudad de México, Facultad Latinoamericana de Ciencias Sociales (FLACSO).

Maron, N. y Connel, J., 2008, "Back to Nukunuku: Employment, Identity and Return Migration in Tonga", en Asia Pacific Viewpoint, 49(2), 168-184. Disponible en http://onlinelibrary.wiley.com/doi/10.1111/j.1467-8373.2008.00368.x/full

Massey, D. S. y Espinosa, K. E., 1997, "What's driving Mexico U.S. migration? A theoretical and policy analysis", en American Journal of Sociology, 122(4), 939999.

Massey Douglas, S., 2016, Border Patrol Policy. Disponible en http://academicminute.org/2016/06/douglasmassey-princeton-university-us-border-patrol-policy/

Massey, Douglas, Pren, Karen y Durand, Jorge, 2009, "Nuevos escenarios de la migración México-Estados Unidos. Las consecuencias de la guerra antiinmigrante", en Papeles de Población, vol. 15, núm. 61, Toluca, México.

Ramírez García, T. y Aguado Ornelas, D., 2013, “Determinantes de la migración de retorno en México, 2007-2009”, en Conapo, Situación demográfica de México 2013 (pp. 175-190).

Ravenstein, E.G., 1885, “The laws of migration”, en Journal of the Royal Statistical Society, vol. 48. Disponible en https://cla.umn.edu/

Ravenstein, E. G., 1889, “The laws of migration”, en Journal of the Royal Statistical Society, vol. LII. Disponible en https://es.scribd.com/

Richmond, A. H., 1968, "Return migration from Canada to Britain", en Population Studies, 22(2), 263-271. Disponible en www.tandfonline.com/

Richmond, Anthony H., 1983, Explaining Return Migration, disponible en https:// journals.sagepub.com/doi/abs/10.1177/019791838301701S39

Rivera Sánchez, L., 2009, “¿Quiénes son los retornados? Apuntes sobre el Migrante retornado en México contemporáneo", en Ponencia presentada en la IV Reunión del Grupo de Trabajo Migración, Cultura y Políticas del Consejo Latinoamericano de Ciencias Sociales-Clacso: La construcción social del migrante. Reflexiones desde América Latina y El Caribe. Ciudad de Guatemala, Guatemala, 14- 16 de octubre de 2009.

Sjaastad, L. A., 1962, “The costs and returns to human migration", en Journal of Political Economy, 70, 80-93. Disponible en https:/www.jstor.org/stable/1829105 
Factores contextuales de la migración de retorno a los municipios de las Zonas Metropolitanas ... / J.G. GONZÁLEZ y E.A. SANDOVAL

Thomas-Hope, E., 1999, "Return migration to Jamaica and its Development Potential”, en International Migration Review, 37(1), 183-207. Disponible http:// onlinelibrary.wiley.com/

Toren, N., 1976, "Return to Zion: characteristics and motivations of returning emigrants", en Social Forces, 54(3), 546-558. Disponible en https://www.researchgate.net/

\section{RESUMEN CURRICULAR DE LOS AUTORES}

\section{Juan Gabino González Becerril}

Maestro en Estudios de Población por El Colegio de la Frontera Norte. Es profesor-investigador de tiempo completo en el Centro de Investigación y Estudios Avanzados de la Población de la Universidad Autónoma del Estado de México (CIEAP/UAEM) y es director de la revista Papeles de Población. Entre sus publicaciones recientes destacan Migración mexiquense a Estados Unidos: un análisis interdisciplinario, 2012; Encuesta sobre Migración de Mexiquenses a Estados Unidos (EMMEU 2009), 2012, Demografía indigena en el Estado de México, 2013 (coautor); Migración Internacional: voces del sur 2017 (compilador); todas las publicaciones bajo el sello editorial de CIEAP-UAEM.

Dirección electrónica: gonzalezg2012@hotmail.com

\section{Eduardo Andrés Sandoval Forero}

Antropólogo, Maestro en Estudios Latinoamericanos, Doctor en Sociología por la Universidad Nacional Autónoma de México (UNAM). Miembro del Sistema Nacional de Investigadores de México desde 1995 (Nivel II). Profesor invitado de universidades de Estados Unidos, América del Sur, España e Italia. Es profesor del curso Migración y codesarrollo en la Cátedra UNESCO, del posgrado de Estudios para la Paz y el Desarrollo, en la Universitat Jaume I, España. Autor de varios artículos, capítulos y libros sobre migración, diversidad cultural y grupos étnicos. Líder del Cuerpo Académico en Migración Interna e Internacional del CIEAP-UAEM. Dirección electrónica: forerosandoval@gmail.com

Artículo recibido el 17 de septiembre de 2018 y aprobado el 12 de junio de 2019 . 